\title{
Caracterización de soldaduras cuproalumi- notérmica en cañerías de conducción para la protección catódica
}

\author{
Copper-Aluminothermic weld characterization \\ on line pipes for cathodic protection
}

Ana Julieta Nehme ${ }^{1}$, Mónica Zalazar ${ }^{1}$

\author{
${ }^{1}$ Departameto de Mecánica Aplicada, Facultad de Ingeniería, Universidad Nacional del Comahue, Buenos Aires 1400, \\ Patagônia, Neuquén, Argentina. \\ e-mail: monica.zalazar@fain.uncoma.edu.ar \\ e-mail otro autor: julietanehme@hotmail.com
}

\section{RESUMEN}

La soldadura cuproaluminotérmica es un proceso de termofusión, en el que el calor necesario se obtiene por el efecto reductor del aluminio sobre el óxido de cobre que genera una reacción exotérmica de alta temperatura, donde el material de aporte será el cobre fundido y recalentado, resultante de dicha reacción química [1]. Esta técnica de unión es empleada en la industria ferroviaria para la unión de los rieles, en las soldaduras de puesta a tierra [2,3] y durante la protección catódica de cañerías de conducción. En este trabajo se caracteriza la soldadura cuproaluminotérmica para la protección catódica de cañerías de acero API 5L X46 y X52 en dos espesores, modificando la sección del conducto de cobre y la carga aplicada. El objetivo es obtener las condiciones óptimas de unión sin afectar la integridad de la cañería para ello se realizarán ensayos macroscópicos, microscópicos y barridos de microdureza en la zona afectada por calor de la soldadura. Se realizaron un total de 25 probetas considerando además distintas marcas de fundentes y tamaños de crisol. Un área de contacto inadecuada puede producir falta de protección catódica de la cañería mientras que una unión con excesiva difusión de cobre en borde de grano puede producir corrosión acelerada y microfisuras en el metal base $[4,1]$ es por ello que se busca la condición óptima de unión en función del grado de acero y espesor de cañería.

Palabras clave: Cuproaluminotérmica soldadura, protección catódica, unión.

\begin{abstract}
Copper-Aluminothermic weld is a process in which welder is generated by thermofusion. The necessary heat is obtained by the aluminum reducing effect above copper oxide, creating a high temperature exothermic reaction, in which melt and overheated copper is the filler material [1]. This technique is used to joint rails in railway industry, in grounding welds [2,3] and also in linepipes for cathodic protection. In this study copperaluminothermic weld is characterized for API 5L X46 and X52 steel linepipes for cathodic protection, considering two different thickness, changing the copper wire section and the flux amount. The purpose is to obtain the optimum welding conditions, therefore macroscopic and microscopic tests, and microhardness sweep are going to be performed on the heat affected zone. Twenty-five test tubes were realized, taking also into account different flux and gunpowder brands and melting pot sizes. An inadequate contact area can cause lack of pipe cathodic protection as well as an excessive copper diffusion into the grain boundaries can produce accelerated corrosion and microcracks in the base metal [4,1], for that reason is necessary to obtain optimum welding conditions according to the steel grade and pipe thickness.
\end{abstract}

Keywords: Copper-aluminothermic, thermit-weld, cathodic protection weld.

\section{INTRODUCCIÓN}

La soldadura cuproaluminotérmica es un proceso de unión generado por la reacción exotérmica que se produce entre el óxido de cobre y el aluminio. Debido al efecto reductor del aluminio, la reacción da como resultado cobre másóxido de aluminio, que es eliminado en forma de escoria debido a que, por su menor peso 
específico, se sobrepone al cobre no interfiriendo en el cuerpo de la soldadura. Esta soldadura tiene propiedades técnico-económicas que hacen a una solución segura y eficiente para los diversos casos que se presentan dentro del campo ingenieril. El proceso se lleva a cabo dentro de un molde de grafito, llamado crisol, fabricado de acuerdo al tipo de conexión a realizar [5].

Actualmente, la corrosión es la principal causa de falla en tuberías alrededor del mundo, la protección catódica junto con los revestimientos son los métodos de protección más utilizados. Para ello se debe soldar un conductor de cobre al cuerpo de la cañería, el que luego es conectado a una red eléctrica a través de la cual se le suministra energía para contrarrestar la corrosión de la misma [6].

La soldadura cuproaluminotérmica presenta varias ventajas a la hora de la realización de trabajos de protección catódica. Al ser una soldadura, la conexión eléctrica se realiza por fusión de los materiales a unir eliminando los intersticios de aire y, por consecuencia, la posibilidad de corrosión galvánica, contaminación interna, aumento de la resistencia eléctrica de contacto. Además, posee mayor o igual capacidad de transferencia de corriente debido a que la sección transversal de la conexión es mayor a la de los conductores a unir, sumado esto a que no tiene factor de cableado [5, 6].

Como el material de aporte empleado es cobre, no existe riesgo de corrosión galvánica por potenciales naturales o por corrientes erráticas. No requiere mano de obra especializada y los tiempos de implementación son bajos. Solo requiere de un molde de grafito y las cargas necesarias para las conexiones. Este equipo es de fácil transporte en la valija de herramientas del electricista. Como el proceso de soldadura es autónomo por el principio de la autofusión [5,6]. Otro factor importante es el hecho de que este procedimiento de unión no requiere de fuentes externas de energía.

En este trabajo presentamos los resultados obtenidos de la realización de soldaduras con distintos parámetros, se caracterizaron las mismas mediante inspección visual, mediciones eléctricas, macrografía, micrografía y medición de microdureza, no se logró en esta etapa del trabajo una buena calidad en la soldadura final por lo que se establecen las condiciones a modificar para mejorar la calidad de las mismas.

\section{MATERIALES Y MÉTODOS}

\subsection{Realización de las soldaduras}

Las soldaduras fueron realizadas sobre cañerías de acero API 5L X46 y X52 en espesores 6,35 mm y 5,56 $\mathrm{mm}$ respectivamente. Se emplearon cables de cobre de $35 \mathrm{~mm}^{2}$ y $4 \mathrm{~mm}^{2}$ de sección. Se trabajó con dos marcas de cargas y de fundentes y se modificó la cantidad de pólvora. Los crisoles fueron del diámetro del cable de cobre. Se realizaron un total de 25 probetas, tanto la preparación del metal base como la soldadura la realizaron técnicos habituados a realizar estas soldaduras.

El procedimientode realización de la soldadura cuproaluminotérmica constó de los siguientes pasos: en primer lugar, se preparó la superficie mediante una lija de grado 80 hasta lograr que la misma quedara brillante. Mediante ultrasonido se verificó que el espesor de la zona a donde se iba a realizar la soldadura tuviera un espesor adecuado (mayor a $5,5 \mathrm{~mm}$ ) y se limpió la superficie con acetona para evitar la presencia de impurezas. Luego, se seleccionó el crisol a utilizar, se mezcló el fundente en su envase (agitación), se colocó la retención en el crisol, seguido del fundente, la pólvora y por último la mecha. Se procedió con el cerrado del crisol y al encendido de la mecha para iniciar la reacción química. La Figura 1 muestra imágenes de esta tarea.

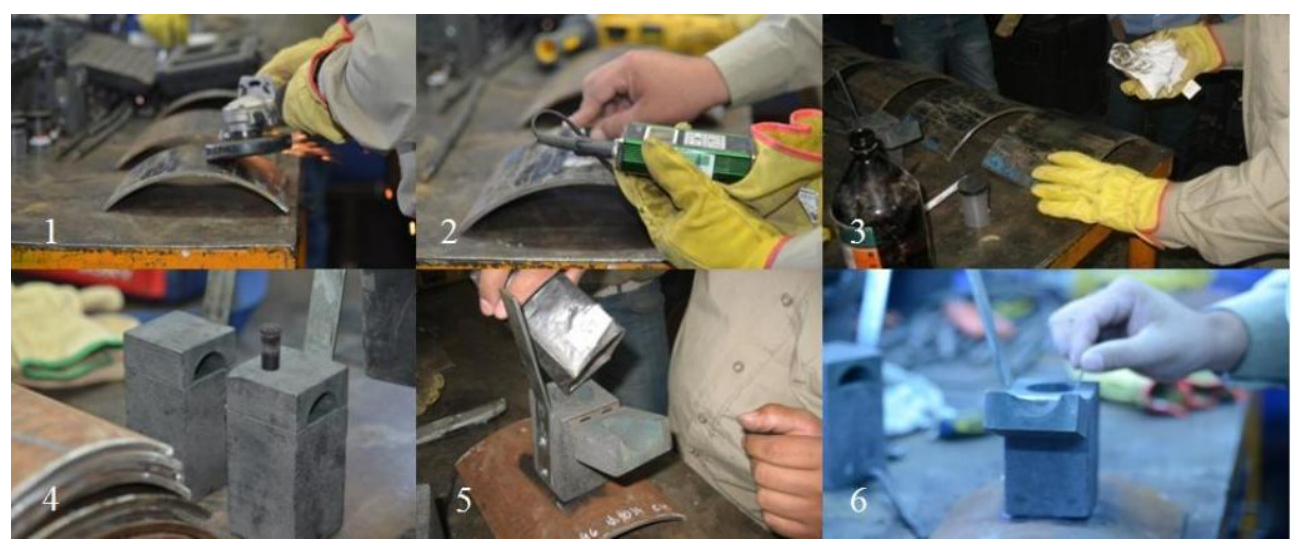

Figura 1: Etapas en la realización de las soldaduras. 1: amolado; 2: medición de espesores; 3: limpieza; 4: selección del crisol; 5: llenado del crisol; 6: encendido. 


\subsection{Caracterización visual de las soldaduras}

A partir de las muestras obtenidas se realizó un examen visual de las mismas. En esta etapa se evaluó si la soldadura presentaba adherencia a la superficie metálica en forma total, parcial (parte de la soldadura continúa adherida a la superficie de la cañería, pero se desprende el cable de cobre) o ninguna(toda la soldadura se desprende de la superficie de acero). Se evaluó además la porosidad y la presencia de teñido térmico en la parte interna de la cañería.

Se efectuaron mediciones eléctricas con el fin de obtener una relación entre la conductividad eléctrica de la soldadura y la calidad de la misma. Se realizó la medición de la resistencia generada teniendo en cuenta que, mientras mejor sea la calidad de la soldadura, la resistencia deberá ser más baja. En bibliografía, se reportan valores de resistencia alrededor de $90 \mu \Omega[4]$, pero no se especifican valores admitidos. Como no se cuentan con valores específicos para la resistencia de estas soldaduras, el ensayo se realizó a modo cualitativo, es decir, no esperando algún valor específico de resistencia para cada soldadura, sino comparando los valores obtenidos entre las mismas. El trabajo fue realizado en el Laboratorio de Mediciones Eléctricas de la Facultad de Ingeniería de la Universidad Nacional del Comahue.

Debido a que los valores de resistencia eléctrica de las soldaduras son muy bajos, y como no se contaba con el equipamiento necesario para la medición directa, se realizaron mediciones indirectas de resistencia. Para ello se utilizó un circuito eléctrico de medición, con el que se hizo circular una corriente conocida y constante a través de cada soldadura y, midiendo la caída de potencial a través de la misma con un voltímetro, se pudo calcular la resistencia de la unión soldada mediante Ley de Ohm.Se introdujo en el circuito una resistencia de $5 \Omega$ (resistencia limitante) para que la corriente en el circuito no dañe la fuente $u$ otros elementos del mismo [7, 8]. Para evitar lecturas erróneas debido a la resistencia propia de los cables de conexión, la medición del voltaje se realizó directamente sobre la soldadura

\subsection{Caracterización metalográfica de las soldaduras}

Posteriormente se prepararonlas probetas metalográficas, para lo cual se cortaron transversalmente en la zona media de cadasoldadura, las muestras obtenidas se incluyeronen una resina acrílica, se realizó un pulido con lijas al agua de granulometría decreciente y finalmente se pulieron con pasta diamantada de $1 \mu \mathrm{m}$. Se realizó una inspección macroscópica para determinar lo siguiente: la presencia de unión metalúrgica, la porosidad y la fusión del cable de cobre. La Figura 2 muestra una probeta preparada (la misma presenta cables de cobre sin fundir).
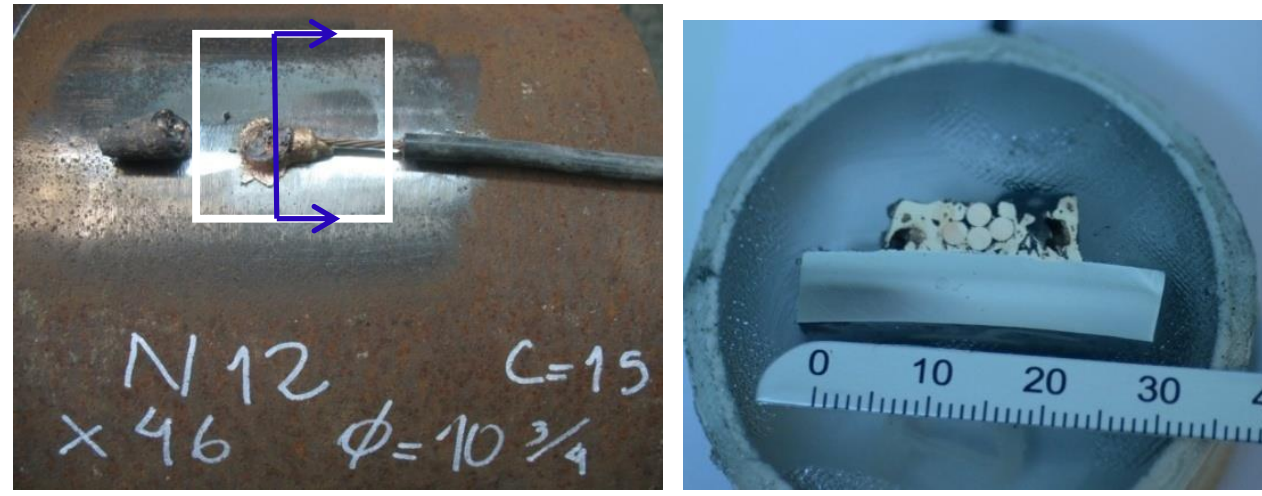

Figura 2: Detalle de la extracción de las probetas y una probeta preparada.

Con las muestras obtenidas y partir del ataque químico realizado con Nital4\% se logró llevar a cabo la inspección microscópica de las soldaduras, permitiendo evidenciar la presencia de impurezas y verificando si existió o no unión metálica entre el cobre y el acero.

Finalmente, se realizaron las mediciones de microdurezaVickers con una carga de $1 \mathrm{Kg}(\mathrm{kg}) \mathrm{y}$ un tiempo de 10 segundos de acuerdo con la norma ASTM E384-10 [9]. Las mediciones se efectuaron sobre el metal base, bordeando al cobre, como se muestra en la Figura 3. Se mantuvo una distancia de 0,5 mm entre cada impronta, excepto en algunos casos en donde se evidenciaba algún defecto sobre el acero que podría llegar a afectar los resultados de la medición. 


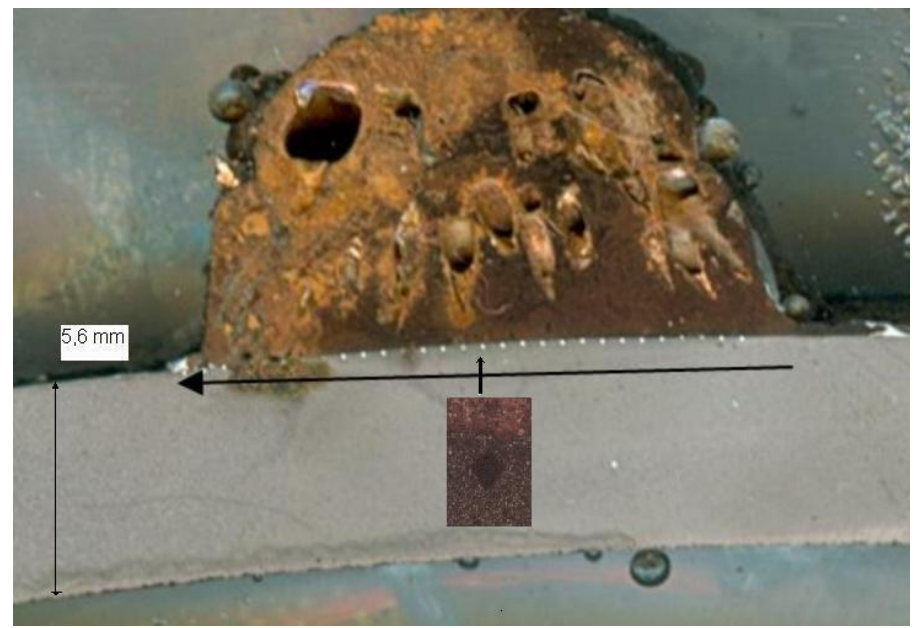

Figura 3: Medición de microdureza.

\section{RESULTADOS Y DISCUSIÓN}

Durante la inspección visual de las muestras obtenidas se descartaron varias soldaduras debido al desprendimiento parcial o total de la misma. En la mayoría de los casos se observó la presencia de teñido térmico en la parte inferior de la cañería y una abundante porosidad superficial.

La Tabla 1 muestra las probetas preparadas y los motivos de la no aceptación de las mismas, de las 25 probetas solo 5 pasaron a la etapa siguiente.

Se realizó el caculo de laresistencia eléctrica de todas aquellas soldaduras que no se descartaron en la primera instancia. Los valores obtenidos fueron fluctuantes, pero la mayoría resultaron mayores a los $100 \mu \Omega$, valor más alto reportado en la bibliografía[9].Aun así, este valor es lo suficientemente bajo para permitir una buena conducción eléctrica, la Tabla 2 muestra los resultados obtenidos, se indica en la Tabla el error en la medición.

Cuando se procediócon el preparado de las probetas, otras muestras fueron descartadas debido al desprendimiento de la soldadura durante el corte metalográfico. Una vez incluidas y preparadas se seleccionaron para el estudio aquellas muestras que presentaron una porosidad no tan excesiva (en todos los casos la porosidad fue elevada, pero en algunos casos más que en otros), donde se pudo observar la fusión del cable de cobre en la soldadura y una unión metálicaconsiderable. Al final del proceso, sólo se continuó el estudio con cinco muestras.

Tabla 1: Muestras obtenidas y resultados de la inspección.

\begin{tabular}{l|l|l|l|l|l|l|l}
\hline $\begin{array}{l}\text { IDENTFI- } \\
\text { CACION }\end{array}$ & $\begin{array}{l}\text { ACERO } \\
\text { API 5L }\end{array}$ & $\begin{array}{l}\text { SECCION } \\
\text { DEL CA- } \\
\text { BLE } \\
\left(\mathbf{m m}^{2}\right)\end{array}$ & $\begin{array}{l}\text { CARGA } \\
\mathbf{( g )}\end{array}$ & $\begin{array}{l}\text { TAMAÑO } \\
\text { CRISOL }\end{array}$ & $\begin{array}{l}\text { PESO } \\
\text { POLVO- } \\
\text { RA } \mathbf{g})\end{array}$ & $\begin{array}{l}\text { MARCA } \\
\text { FUDNEN- } \\
\text { TE }\end{array}$ & RESULTADOS DE LA VISUAL \\
\hline M1 & X52 & 35 & 15 & 35 & 2 & FACBSA & Desprendimiento parcial \\
\hline M2 & X52 & 35 & 45 & 35 & 2 & FACBSA & $\begin{array}{l}\text { Sin desprendimiento. No se fundió } \\
\text { el cable. Abundante porosidad. }\end{array}$ \\
\hline M3 & X52 & 35 & 15 & 35 & 2 & FACBSA & Desprendimiento total. \\
\hline M4 & X52 & 35 & 45 & 35 & 2 & FACBSA & $\begin{array}{l}\text { Sin desprendimiento. No se fundió } \\
\text { el cable. }\end{array}$ \\
\hline M5 & X52 & 35 & 45 & 35 & 2 & FACBSA & $\begin{array}{l}\text { Sin desprendimiento. No se fundió } \\
\text { el cable. Abundante escoria. }\end{array}$ \\
\hline M6 & X52 & 35 & 45 & 35 & 2 & FACBSA & $\begin{array}{l}\text { Sin desprendimiento. No se fundió } \\
\text { el cable. }\end{array}$ \\
\hline M7 & X52 & 35 & 15 & 30 & 3 & FACBSA & Desprendimiento total. \\
\hline
\end{tabular}




\begin{tabular}{|c|c|c|c|c|c|c|c|}
\hline M8 & X52 & 35 & 15 & 30 & 3 & FACBSA & $\begin{array}{l}\text { Sin desprendimiento. No se fundió } \\
\text { el cable. }\end{array}$ \\
\hline M9 & $\mathrm{X} 52$ & 35 & 15 & 30 & 3 & FACBSA & Desprendimiento parcial. \\
\hline M10 & $\mathrm{X} 46$ & 4 & 15 & 45 & 2 & FACBSA & $\begin{array}{l}\text { Sin desprendimiento. No se fundió } \\
\text { el cable. }\end{array}$ \\
\hline M11 & X46 & 4 & 15 & 45 & 2 & FACBSA & $\begin{array}{l}\text { Hay unión metalúrgica. Abundante } \\
\text { porosidad. }\end{array}$ \\
\hline M12 & X46 & 4 & 15 & 45 & 2 & FACBSA & Desprendimiento parcial. \\
\hline M13 & $\mathrm{X} 46$ & 4 & 45 & 35 & 1,88 & GENROD & Desprendimiento total. \\
\hline M14 & $\mathrm{X} 46$ & 35 & 30 & 35 & 2,34 & GENROD & Desprendimiento parcial. \\
\hline M15 & $\mathrm{X} 46$ & 4 & 15 & 45 & 1,98 & FACBSA & $\begin{array}{l}\text { Poca unión metalúrgica. Abundante } \\
\text { porosidad. }\end{array}$ \\
\hline M16 & $\mathrm{X} 46$ & 4 & 30 & 35 & 2,30 & FACBSA & $\begin{array}{l}\text { Buena unión metalúrgica. Abun- } \\
\text { dante porosidad hacia la superficie. }\end{array}$ \\
\hline M17 & $\mathrm{X} 46$ & 4 & 20 & 35 & 1,68 & POWER & $\begin{array}{l}\text { Buena unión metalúrgica. Abun- } \\
\text { dante porosidad hacia la superficie. }\end{array}$ \\
\hline M18 & $\mathrm{X} 46$ & 4 & 25 & 35 & 1,78 & POWER & $\begin{array}{l}\text { Sin desprendimiento. Abundante } \\
\text { porosidad. }\end{array}$ \\
\hline M19 & $\mathrm{X} 46$ & 4 & 15 & 45 & 2,05 & FACBSA & Desprendimiento parcial. \\
\hline M20 & $\mathrm{X} 46$ & 35 & 30 & 35 & 2,4 & POWER & $\begin{array}{l}\text { Sin desprendimiento. No se fundió } \\
\text { el cable. }\end{array}$ \\
\hline M21 & $\mathrm{X} 46$ & 35 & 30 & 35 & 1,92 & FACBSA & $\begin{array}{l}\text { Sin desprendimiento. No se fundió } \\
\text { el cable. }\end{array}$ \\
\hline M22 & $\mathrm{X} 46$ & 4 & 30 & 35 & 1,88 & FACBSA & Desprendimiento parcial. \\
\hline M23 & $\mathrm{X} 46$ & 4 & 30 & 35 & 2,29 & FACBSA & Desprendimiento parcial. \\
\hline M24 & $\mathrm{X} 52$ & 4 & 25 & 25 & 1,74 & FACBSA & $\begin{array}{l}\text { Buena unión metalúrgica. Abun- } \\
\text { dante porosidad hacia la superficie. }\end{array}$ \\
\hline M25 & X52 & 4 & 25 & 20 & 2,05 & FACBSA & Desprendimiento parcial. \\
\hline
\end{tabular}

Tabla 2: Valores de las resistencias de las soldaduras para las muestras obtenidas que siguieron siendo analizadas.

\begin{tabular}{l|l|l}
\hline $\begin{array}{l}\text { MUES- } \\
\text { TRA }\end{array}$ & $\begin{array}{l}\mathbf{R}_{\text {SOldadura }} \\
(\mathbf{m} \boldsymbol{\Omega})\end{array}$ & $\Delta \mathbf{R}(\mathbf{m} \boldsymbol{\Omega})$ \\
\hline M11 & 0,128 & 0,039 \\
\hline M16 & 0,103 & 0,039 \\
\hline M17 & 0,051 & 0,039 \\
\hline M18 & 0,292 & 0,041 \\
\hline M24 & 0,087 & 0,039 \\
\hline
\end{tabular}

Como se puede observar, tres de los valores resultaron superiores a $100 \mu \Omega$, en especial para la muestra M18. Los valores más bajos se obtuvieron para las muestras M17 y M24, sin embargo, los valores reportados en bibliografía se encuentran alrededor de los $90 \mu \Omega$. Los elevados valores de resistencia se deben a la porosidad en las soldaduras realizadas.

El estudio continuó con el análisis micrográfico, para lo cual se atacaron las muestras seleccionadas con Nital 4\%. En ninguno de los casos se observó la presencia de martensita en la zona afectada por calor (ZAC). En todos los casos la porosidad fue alta, pero se detectaron zonas con presencia de unión metalúrgica y penetración adecuada del cobre en los bordes de grano del metal base. Solo una de las muestras evidenció falta de unión metalúrgica por presencia de escorias. La Figura 4 muestra la micrografia obtenida en la muestra M17. 

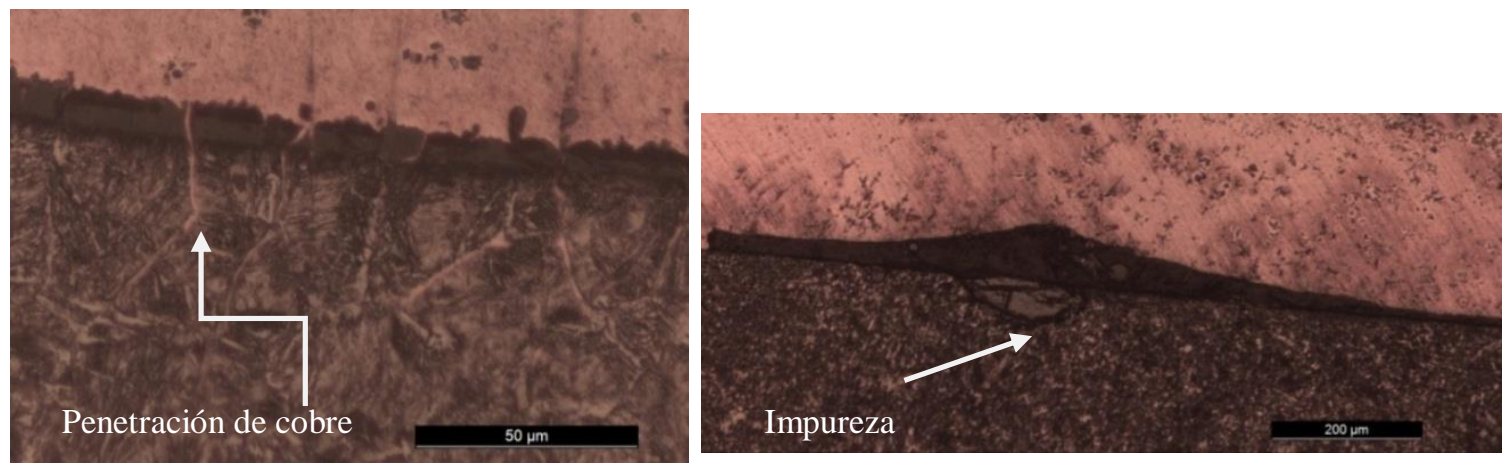

Figura 4: Microestructura soldadura cuproaluminotérmica Muestra M17.

El análisis de microdureza mostró en la soldadura del acero API 5LX52 (M24) los menores valores con caídas importantes de dureza en la ZAC. En las soldaduras del acero API 5LX46,en dos de las muestras se observaron durezas altas por encima de HV1 320 resultados aleatorios para las distintas soldaduras cuproaluminotérmicas realizadas, demostrando que no en todos los casos el grado de unión metálica fue el mismo y variando considerablemente debido a que varias regiones en una misma muestra mostraron zonas sin unión. En la Figura 5 se observan los resultados obtenidos.

A partir de la variabilidad en los resultados, y considerando que en ninguno de los casos el resultado fue óptimo, se optó por proponer un nuevo protocolo de soldadura. En todos los ejemplares la soldadura resultó muy porosa y se consideró a la misma como resultado de la humedad que pudo llegar a absorber el grafito del ambiente, por lo cual, a los pasos anteriores para la realización de la soldadura se le adicionó uno intermedio que involucra el secado del crisol con una pistola de calor, de modo de poder eliminar este factor. También se procedió a secar el fundente en un horno eléctrico con el mismo fin.

Se observó que en la realización de la soldadura resulta imprescindible la limpieza de la superficie a soldar. La escoria produce una unión deficiente del cobre con la superficie de la cañería.

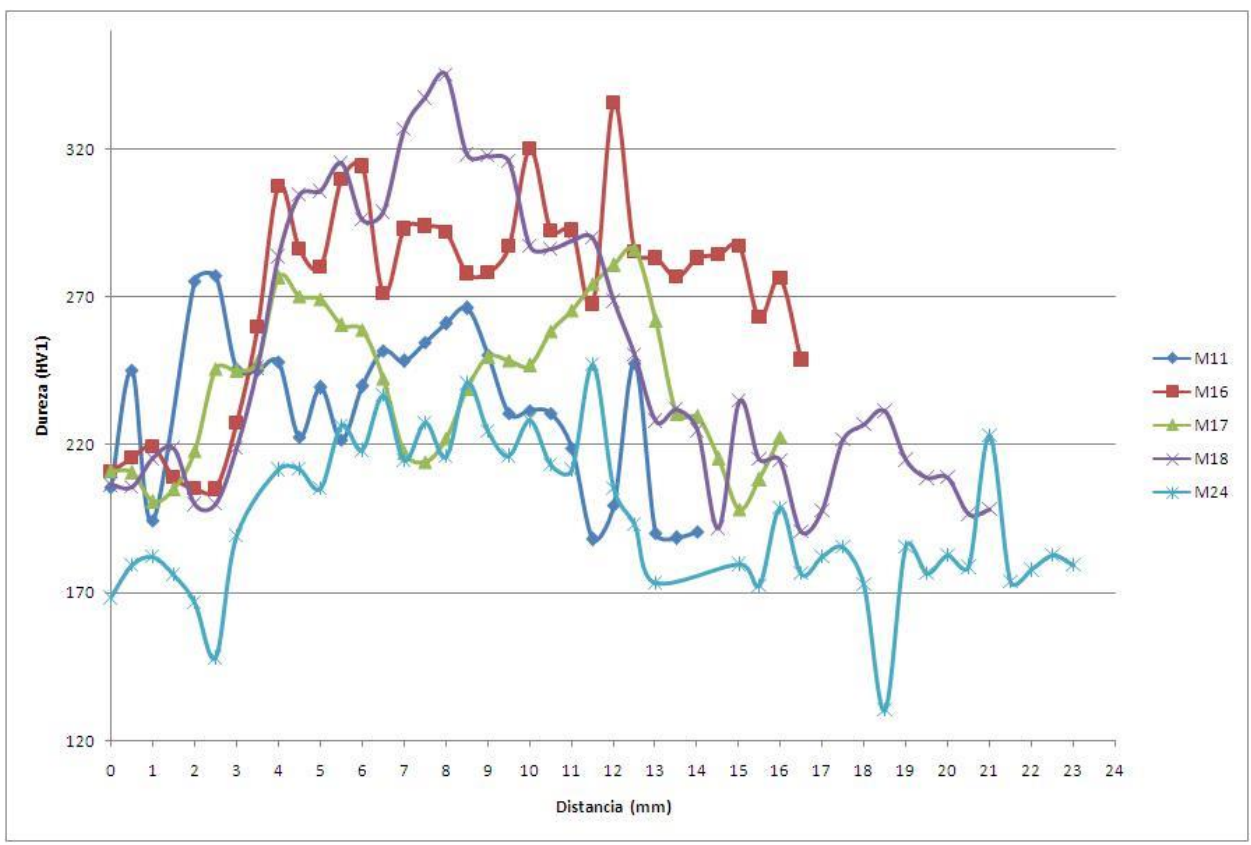

Figura 5: Resultados del barrido de microdureza. 


\section{CONCLUSIONES}

Se observó que el conductor de $35 \mathrm{~mm}^{2}$ no permite obtener, para el crisol utilizado, buena unión metalúrgica.El cable de cobre de $4 \mathrm{~mm}^{2}$ de sección mostró buen comportamiento. Los resultados arrojados a lo largo de estudio fueron muy variables y el único patrón que se logró obtener a partir de las muestras que pasaron la etapa de selección, fue que, para las condiciones en las que la soldadura fue realizada, el cable de cobre de 4 $\mathrm{mm}^{2}$ fue apropiado.

\section{AGRADECIMIENTOS}

A la empresa y técnicos que realizaron las soldadura. Al personal del Laboratorio de mediciones eléctricas de esta Facultad y a la Secretaria de Investigación de la UNCo por financiar el proyecto PROIN 04-I194.

\section{BIBLIOGRAFÍA}

[1] WELDING HANDBOOK -Materials and applications Part 2, 8 ed., Editorial AWS, 1998.

[2] ARCIONI, J.C, GIMENEZ, J.F. "Las soldaduras cuproaluminotérmicas para puesta a tierra y sus ensayos eléctricos de calentamiento", Revista electrotécnica, v. 274, pp. 86-91, Mar2013.

[3] NORMA IRAM 2315*; Materiales para puesta a tierra - Soldaduracuproaluminotérmica, 2 ed., 1999.

[4] LE DUC, E., LO CASTRO, B, LAVINAUD, B. "Method of Aluminothermic Welds Qualification for Down Conductors and Earth-Terminations Systems", In:International Symposium on Lightning Protection, (XII SIPDA), pp. 345-347, Belo Horizonte, Brazil, October 7-11, 2013.

[5] CUPROTEC, http://www.cuprotec.com.ar/. Accedido en Junio de 2016.

[6] FASTEN, http://www.fasten.com.ar/. AccedidoenJunio de 2016.

[7] WENNER, F., A method of measuring earth resistivity,v. 12, Washington, D.C., U.S. Dept. of Commerce, Bureau of Standards, 1916.

[8] ZBAR, MALVINO, MILLER, Prácticas de Electrónica, Marcombo, BoixareuEditores, 2001.

[9] ASTM E 384-10, Standard Test Method for Miroindentation Hardness of Materiales, West Conshohocken, United States ASTM, International, 100 Barr Harbor Drive, PO Box C700, 2007. 